\title{
Preferential concentration and settling of heavy particles in homogeneous turbulence
}
A. Dejoan and R. Monchaux

Citation: Phys. Fluids 25, 013301 (2013); doi: 10.1063/1.4774339

View online: http://dx.doi.org/10.1063/1.4774339

View Table of Contents: http://pof.aip.org/resource/1/PHFLE6/v25/i1

Published by the American Institute of Physics.

\section{Related Articles}

Intermittency and local Reynolds number in Navier-Stokes turbulence: A cross-over scale in the Caffarelli-KohnNirenberg integral

Phys. Fluids 24, 115112 (2012)

A modified nonlinear sub-grid scale model for large eddy simulation with application to rotating turbulent channel flows

Phys. Fluids 24, 075113 (2012)

Scaling range of velocity and passive scalar spectra in grid turbulence

Phys. Fluids 24, 075101 (2012)

On Lagrangian single-particle statistics

Phys. Fluids 24, 055102 (2012)

The length distribution of streamline segments in homogeneous isotropic decaying turbulence

Phys. Fluids 24, 045104 (2012)

\section{Additional information on Phys. Fluids}

Journal Homepage: http://pof.aip.org/

Journal Information: http://pof.aip.org/about/about_the_journal

Top downloads: http://pof.aip.org/features/most_downloaded

Information for Authors: http://pof.aip.org/authors

\section{ADVERTISEMENT}

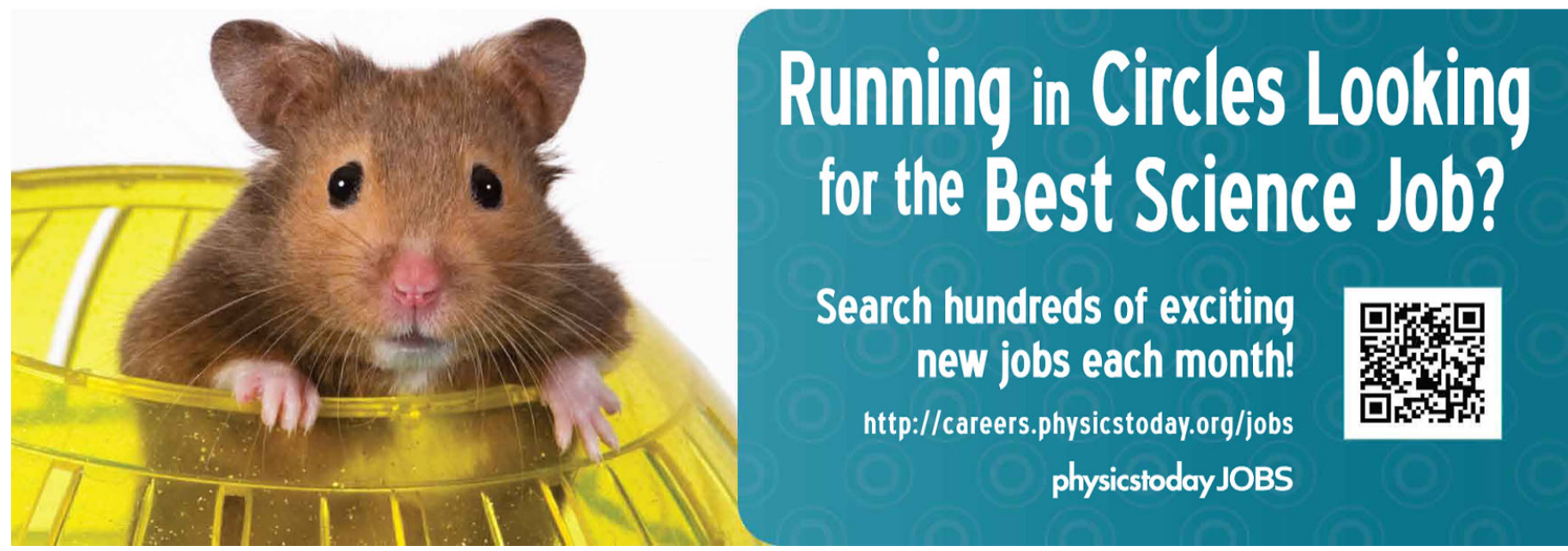




\title{
Preferential concentration and settling of heavy particles in homogeneous turbulence
}

\author{
A. Dejoan ${ }^{1}$ and R. Monchaux ${ }^{2}$ \\ ${ }^{1}$ Unidad de Modelización y Simulación de Procesos Centro de Investigaciónes Energéticas \\ Medioambientales y Tecnológicas (CIEMAT), Av. Complutense, 28040 Madrid, Spain \\ ${ }^{2}$ Unité de mécanique, Ecole Nationales Supérieure de Techniques Avancées, ParisTech, \\ Chemin de la Hunière, 91761 Palaiseau Cedex, France
}

(Received 28 May 2012; accepted 28 November 2012; published online 16 January 2013)

\begin{abstract}
Voronoï diagrams are used to analyze one-way coupling direct numerical simulation data of heavy particles settling in homogeneous turbulence. Preferential concentration and clustering of the inertial particles are analyzed for an extended range of particle Stokes and Rouse numbers. Influence of preferential concentration on the settling velocity enhancement is addressed from statistics of particle and flow field quantities conditioned on the local concentration. While gravity is found to have almost no influence on the global characteristics of preferential concentration, the conditional statistics bring out a refined preferential sampling of the flow field resulting from the gravitational effects. This preferential sampling shows that beside the descending fluid velocity contribution, the settling velocity is further increased by the descending fluid acceleration. This effect cannot be detected from global estimations of the particle concentration field. A 2D analysis of the Voronoï cells is also presented to investigate their shape and orientation. It is found that clusters can be represented as 2D elongated manifolds. Their shape is shown to be similar in zero and non-zero gravity fields while Voronoï cells tend to be more elongated for Stokes numbers around unity. Under the gravity effects, they tend to be preferentially oriented perpendicularly to the gravitational axis. (c) 2013 American Institute of Physics. [http://dx.doi.org/10.1063/1.4774339]
\end{abstract}

\section{INTRODUCTION}

Transport of inertial particles in turbulent flows has received a growing interest in the last decades. Because of its relevance in environmental problems (pollutant or dust dispersion in the atmosphere, rain drop formation) and engineering processes (spray combustion in diesel engines), the preferential concentration of particles much heavier than the surrounding flow has been the focus of many experimental and numerical studies. ${ }^{6,19,20}$ Still, it is a largely open question and some of its effects such as the increase of the particle collision rate ${ }^{3,16,18}$ and of the settling velocity $2,22,25,26$ are not yet fully understood.

In this article, we address the concentration and the settling issues by making use of direct numerical simulations (DNS) in the so-called "one-way coupling" regime where no back reaction is exerted by the particles on the carrier fluid. As a result of their inertia, particle dynamics lags behind the fluid's one. This effect is characterized by the Stokes number which, in a turbulent flow, is defined as $S t=\tau_{p} / \tau_{\eta}$, the ratio between the particle viscous response time $\tau_{p}$ and the Kolmogorov viscous time $\tau_{\eta}$. In a quiescent fluid and under gravity, a particle settles and reaches a steady velocity $v_{t}$, referred to as its terminal velocity. In a turbulent flow, the settling velocity of heavy particles has been observed to be larger than $v_{t}$. Former numerical studies ${ }^{22,24,25}$ explain this settling enhancement by the inertial bias mechanism, responsible of the migration of heavy particles to the periphery of turbulent vortical structures in zero-gravity conditions and which, under gravity effects, preferentially sample the side of descending fluid motions of such structures. Experimental results are scarce due to the difficulties encountered in obtaining an accurate measure of the particle vertical velocity. 
Nevertheless, Aliseda et al. ${ }^{2}$ have evidenced that mass loading effects and local concentration do influence the settling velocity enhancement. These concentration effects have not been much studied so far, neither the influence of gravity on the preferential concentration. The present study aims to contribute to bridging this gap. For this purpose, we propose to apply the Voronoï diagrams analysis ${ }^{13,14,21}$ to data obtained from DNS of heavy particles settling in statistically stationary homogeneous and isotropic turbulence. Different particle inertia and gravity fields are considered in order to cover a range of Stokes and Rouse number as large as possible, the Rouse number being defined as $R=\left|v_{t} / u^{\prime}\right|$, where $u^{\prime}$ denotes the root mean square of the fluctuating turbulent velocity. Note that the one-way coupling approach chosen here only allows to address local concentration effects. To undertake mass loading effects a two-way coupling approach is required, this will be considered in a next study.

The paper is organized as follows: in Sec. II we present the numerical simulation methodology and parameters (Sec. II A) and the post-processing principles, including the basics of the Voronoï analysis (Sec. II B). Our results are given in Sec. III where we successively address the influence of gravity on preferential concentration (Sec. III A) and the influence of local concentration and gravity on the settling velocity enhancement (Sec. III B). Section III C presents some relevant conditional statistics of the fluid phase fields in order to get more insight in the results given in the preceding sections. We finally discuss these results and draw conclusions in Sec. IV.

\section{NUMERICAL SIMULATIONS AND POST-PROCESSING}

\section{A. Numerical simulations}

The homogeneous and isotropic turbulence is described in the Eulerian frame by the incompressible Navier-Stokes equations

$$
\begin{gathered}
\frac{\partial u_{i}}{\partial t}+u_{j} \frac{\partial u_{i}}{\partial x_{j}}=-\frac{1}{\rho} \frac{\partial p}{\partial x_{i}}+v \frac{\partial^{2} u_{i}}{\partial x_{j} \partial x_{j}}+f_{i}, \\
\frac{\partial u_{i}}{\partial x_{i}}=0,
\end{gathered}
$$

where $i=1,2,3$ refers to the three cartesian directions (gravity is along the third one denoted $z$ in the following), $x_{i}$ is the spatial coordinates, $u_{i}$ is the fluid velocity, $\rho$ is the density, $p$ is the pressure, and $v$ is the kinematic viscosity. The statistically steady turbulence is achieved through the external energy source term, $f_{i}$, that injects energy at low wavenumbers such that turbulence energy dissipation is balanced. The particles, with density $\rho_{p}$ much larger than the fluid density $\rho$, are described in the Lagrangian frame by a simplified version of the equation of motion of Maxey, Riley, and Gatignol: ${ }^{7,10}$

$$
\frac{d v_{i}}{d t}=\frac{\left(u_{x_{p_{i}}}-v_{i}\right)}{\tau_{p}}+\frac{v_{t_{i}}}{\tau_{p}}
$$

where $v_{i}$ is the particle velocity, $u_{x_{p_{i}}}$ is the instantaneous fluid velocity at the particle location $x_{p_{i}}$ and $m_{p}$ the particle mass. The response time of the particles, $\tau_{p}$, is given by $\tau_{p}=d^{2} \rho_{p} /(18 v \rho)$ with $d$ the particle diameter; $v_{t_{i}}$ is the terminal velocity in the still fluid given by $v_{t_{i}}=\tau_{p} g_{i}\left(1-\rho / \rho_{p}\right)$ where $g_{i}$ is the gravitational acceleration component. Following, we will denote the settling velocity as $v_{t}$. By defining $\vec{x}, \vec{y}$, and $\vec{z}$ as the unit vectors along each direction, the gravity vector is given by $-|g| \vec{z}$ where the magnitude of the acceleration $|g|$ is adjusted accordingly to $v_{t}$ and $\tau_{p}$.

The Navier-Stokes equations are solved on a cubic fluid box of side length $L_{\mathrm{box}}=2 \pi$, discretized into $N^{3}$ computational nodes, with periodic boundary conditions. In the three spatial directions of the computational domain a pseudo-spectral algorithm with a de-aliasing truncation technique (referred to as the " $2 / 3$ rule") is used with a second-order Runge-Kutta time-stepping for the nonlinear terms and an analytic integrating factor for the viscous terms. The forcing is realized by distributing the power input $f_{i}$ over a narrow band of wavenumbers $k$ that satisfy $k_{p}-1 \leq k \leq k_{p}$ +1 , where $k_{p}$ defines the peak forcing mode (see Refs. 9 and 17 for further computational details). The main numerical and flow parameters are provided in Table I. The value of the microscale Reynolds number $R_{\lambda}=40$ is moderate but was shown to be large enough to properly simulate 
TABLE I. Numerical and flow parameters. Microscale Reynolds number $R_{\lambda}$, number of computational nodes $N^{3}$, viscosity $v$, box side length $L_{\text {box }}$, integral length scale $L$ given by $\frac{\pi}{2 u^{\prime 2}} \int \frac{E(k)}{k} d k$ (where $u^{\prime}$ is the fluctuating velocity defined by $u^{\prime 2}=\frac{2}{3} \int E(k) d k$ with $E(k)$ the energy spectrum), large-eddy turn-over time $T$ given by $\frac{L}{u^{\prime}}$, Kolmogorov length and time scales $\eta$ and $t_{\eta}$, maximum wavenumber $k_{\max }=\sqrt{2} N / 3$ and forcing mode $k_{p}$.

\begin{tabular}{cccccccc}
\hline \hline$R_{\lambda}$ & $N^{3}$ & $v$ & $L / L_{\text {box }}$ & $L / \eta$ & $T / t_{\eta}$ & $k_{\max } \eta$ & $k_{p}$ \\
\hline 40 & $64^{3}$ & 0.0178 & 0.211 & 31.56 & 15.85 & 1.32 & 3 \\
\hline \hline
\end{tabular}

the turbulent flow required for the present study (see Refs. 22 and 26). This allows to encompass a full set of relevant parameters (particle sample, Stokes and Rouse numbers, see below) while maintaining reasonable computational CPU times.

A fourth-order Lagrangian polynomial interpolation is used to evaluate the fluid velocity at the particle position required for the computation of the drag force exerted by the fluid on the particle. The particles are homogeneously introduced in the fluid once the turbulence shows a statistically stationary state and the statistics of the particle fields are initiated after several integral time scales $(\sim 20 T)$ after their injection. The different values of the gravity field considered in this study are chosen by selecting four values of the Rouse number, $R=0,0.25,0.5$, and 1 . Four values of the Stokes number are also considered: $0.36,1,2$, and 6 . This leads to 16 points in the parameter space. For each case $N_{p}=3.2 \times 10^{5}$ particles are tracked.

\section{B. Post-processing}

This paper focuses on the possible correlation between preferential concentration and settling velocity enhancement. A good knowledge of the particle concentration field at the particle locations is thus required. To achieve this, we make use of Voronoï diagrams. For $N_{p}$ particles evolving in a ND space, these are uniquely defined as the ensemble of the $N_{p}$ polyhedrons associated to each particle and defined as the subset of the ND space that is closer to this particle than to any other. From their definition, the Voronoï volumes are exactly the inverse of the local concentration field calculated at an intrinsic resolution (see Ref. 14 for further details on the relevance of the Voronoï diagrams in this context).

For the 16 cases considered here, we isolate 20 snapshots regularly covering 8 integral time scales. Practical calculations are performed using MATLAB algorithm. In order to get rid of the infinite and non-physical volumes arising at the periphery of the computational cube, we make use of the data periodicity. Consequently, we achieve relevant calculation of the $N_{p}=3.2 \times 10^{5}$ Voronoï volumes associated to the 20 realizations of the particle field.

The average of the Voronoï volumes being trivially equal to $(2 \pi)^{3} / N_{p}$, the Voronoï volumes are normalized to achieve unit mean and are denoted by $\mathcal{V}$. By making use of the statistical stationarity of our flow, we gather the 20 snapshots to achieve larger particle samples. We thus define the overall average operator denoted by an over-bar, ${ }^{-}$, which actually represents a time space average, and the related standard deviation, $\sigma$. .

Following Monchaux et al., ${ }^{13}$ clusters of particles are identified as connected components of Voronoï volumes whose individual volume is below a given threshold. This threshold is simply defined as the intersection of the preferentially concentrated particle PDF of $\mathcal{V}$ and the corresponding PDF obtained from a uniform random distribution of particles.

The fourth-order Lagrangian polynomial interpolation scheme evoked in Sec. II A is also used to evaluate several fields associated with the fluid phase at the particle location (acceleration, OkuboWeiss parameter, strain tensor, vorticity...). Using the local concentration, $C$, measured as the inverse of the normalized Voronoï volume, we present results conditioned on the local concentration. By reference to the average concentration, $C_{0}=N_{p} /(2 \pi)^{3}$, we define the following six ranges of relative concentration: $0 \leq C / C_{0} \leq 0.3,0.3 \leq C / C_{0} \leq 0.6,0.6 \leq C / C_{0} \leq 1,1 \leq C / C_{0} \leq 2,2 \leq C / C_{0} \leq 4$, and $4 \leq C / C_{0}$. We have checked that modifying slightly the values of the upper and lower limits of these bins does not affect the quantitative results. ${ }^{1}$ The values of $C / C_{0}$ appearing in the various figures corresponds to actual average values of $C / C_{0}$ calculated within these bins for each case. We define the average of any quantity $X$ performed over time and particle samples as $\bar{X}$. 

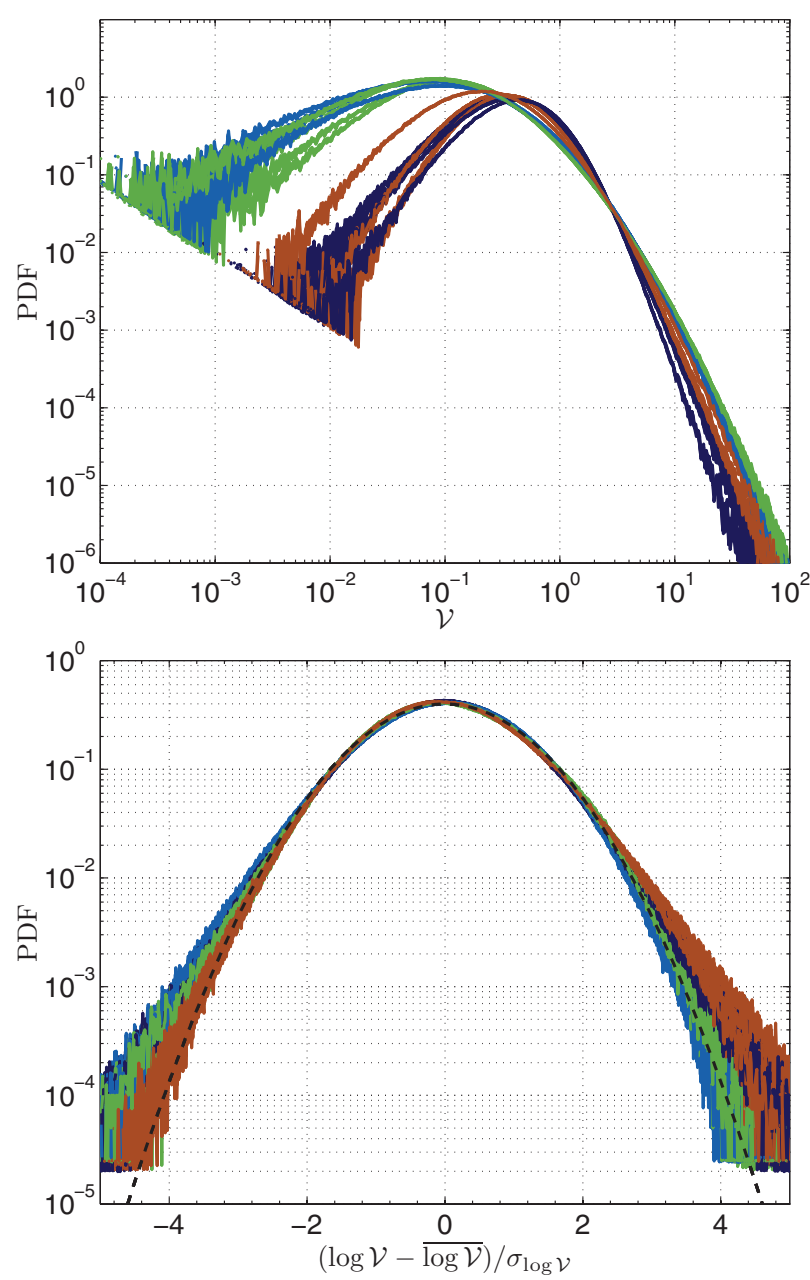

FIG. 1. PDF of normalized Voronoï volume $\mathcal{V}$; Centered and normalized PDF of the logarithm of normalized Voronoï volumes, black dashed line represents a Gaussian distribution. Each figure gathers all Rouse and Stokes number values considered in the simulations. Dark blue: $S t=0.36$, light blue: $S t=1$, green: $S t=2$, and dark orange: $S t=6$.

Beside the 3D Voronoï analysis described above, we have also performed a 2D Voronoï analysis with the aim of studying the orientation of the Voronoï cells. For this, 5 slices of size $2 \pi \times 2 \pi$ $\times 4 \eta$ regularly spaced along the gravity direction $(\vec{z})$ and 5 other similar slices regularly spaced along one of the direction perpendicular to gravity $(\vec{x})$ were extracted from the data. A total of 100 2D slices were used for the statistics. In Ref. 12, such 2D projections were shown to be relevant and to not introduce qualitative nor quantitative biases on the obtained results. Each Voronoï cell being defined by its vertices, we have evaluated the associated moments $\left(\lambda_{1}, \lambda_{2}\right)$ and axis $\left(\vec{e}_{1}, \vec{e}_{2}\right)$ of inertia in the considered plane. The ratio $\lambda_{1} / \lambda_{2}$ characterizes the cell aspect ratio which value is between 0 and 1. Note that $\lambda_{1}$ will denote the smallest eigenvalue of the inertia tensor so that a value of $\lambda_{1} / \lambda_{2}$ different from 1 means that the cell will be elongated along the associated inertia axis $\vec{e}_{1}$. The preferential orientation of the $2 \mathrm{D}$ Voronoï cells is analysed by evaluating the angle between $\vec{e}_{1}$ and the unit vectors of the reference simulation box of the considered plane.

\section{RESULTS}

\section{A. Preferential concentration and clustering}

Figure 1 presents the PDFs of the normalized Voronoï volumes, $\mathcal{V}$, and of its logarithm for the full set of simulation data. Regarding the linear PDFs, it is worth noticing that the right tail is 

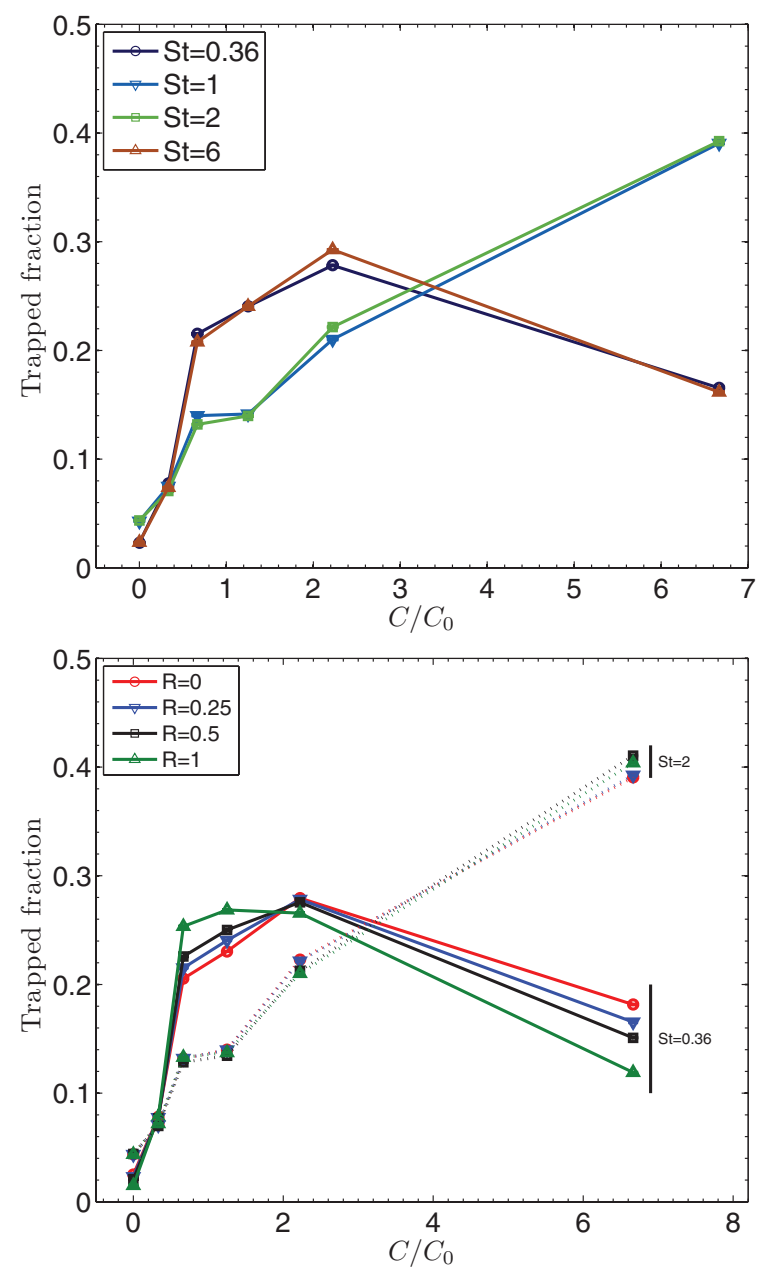

FIG. 2. Particle trapped fraction given as a function of the local concentration. (Top) Fixed gravity such that $v_{t}=v_{\eta}$ $(R=0.25)$ for the four values of the Stokes number. (Bottom) Four values of the Rouse number $(\bigcirc: R=0, \nabla$ : $R=0.25, \square: R=0.5, \triangle: R=1)$ and two values of the Stokes number ( $S t=0.36$ : thick line and $S t=2$ : dashed line).

much less sensitive to changes in Stokes number and/or gravitation strength than the left tail. For any set of the data analysed, the centered-reduced PDFs of $\log (\mathcal{V})$ are close to Gaussian in the range $\pm 2 \sigma$, indicating log-normal distributions of the Voronoï volumes. These PDFs can thus be studied solely from their standard deviation. These results corroborate what has already been observed for experimental data by Monchaux et al. ${ }^{13}$ and more recently for DNS data by Meneguz and Reeks. ${ }^{11}$ Note, however, that a Gamma distribution was found for the PDF shapes by Tagawa et al. ${ }^{21}$ for similar DNS data. Private discussions with the latter authors were not sufficient to understand so far this discrepancy.

In Fig. 3 (top) the standard deviation of the Voronoï volumes $\sigma_{\mathcal{V}}$ is plotted as a function of the Stokes number for all the values of Rouse number considered (note that the error bars associated with the statistical dispersion obtained between 10 subsamples of the original data set are smaller than the symbols in most of the cases). Whatever the value of $R$, the standard deviation is maximal for $S t=2$ consistently with all similar previous studies. Interestingly, the two curves corresponding to the intermediate values of the Rouse number $(R=0.25$ and $R=0.5)$ are almost superimposed while the two others are clearly different even if gravity has clearly less influence on preferential concentration than the Stokes number.

The fraction of particles trapped in a region of a given concentration $C / C_{0}$ is displayed in Fig. 2 . This ratio actually represents the integral of the PDFs displayed in Fig. 1. For $S t=1$ and 2 the fraction of trapped particles monotonically grows with $C / C_{0}$ and the very high concentration regions 

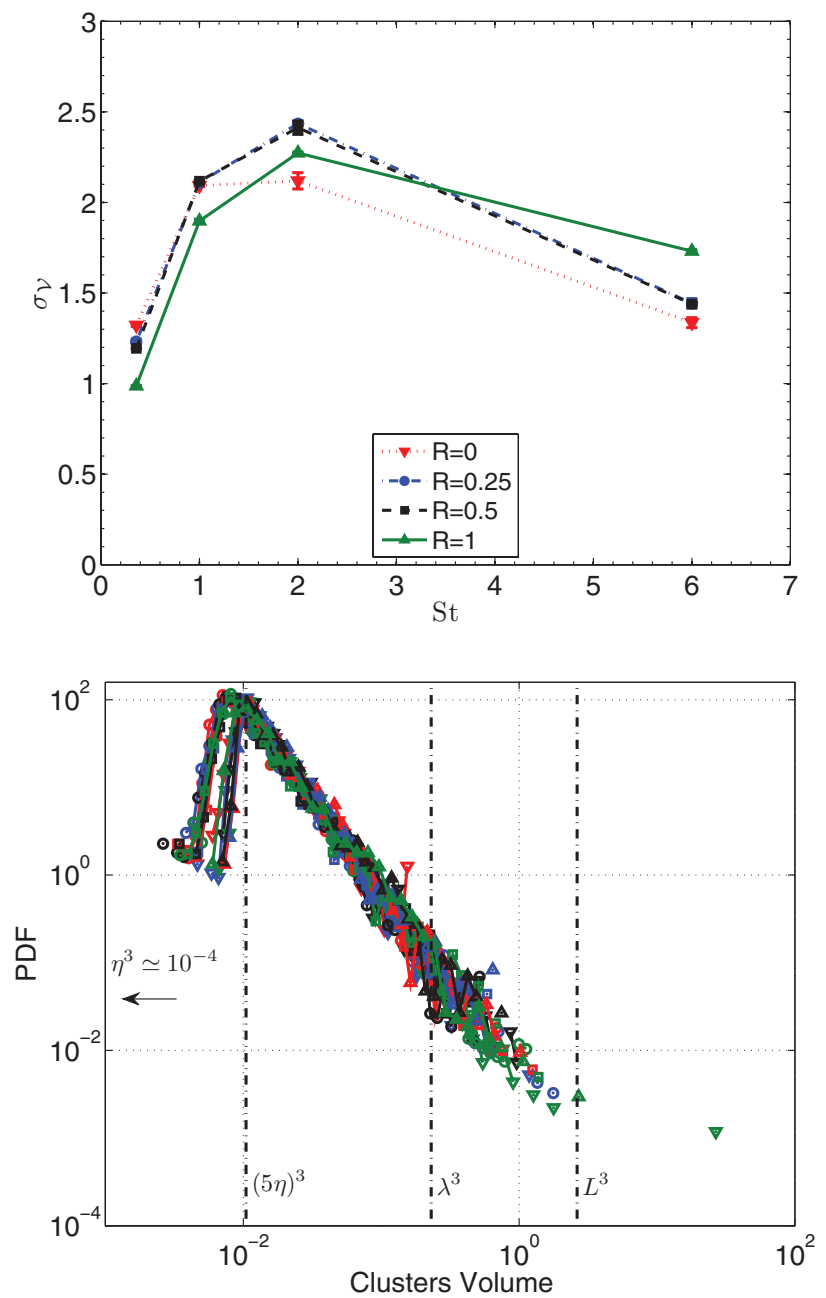

FIG. 3. (Top) Standard deviation of the Voronoï volumes given as a function of the Stokes number for the four values of Rouse number. For a uniform random distribution of particles, this deviation standard is around 0.44. (Bottom) PDF of clusters volumes given for the full values of Rouse and Stokes numbers.

count up to $40 \%$ of the particle sample. The high concentration regions are much more depleted for $S t=0.36$ and $6(\simeq 20 \%)$, a maximum number of particles $(\simeq 30 \%)$ being found at intermediate concentration regions with $C / C_{0} \simeq 2$. The ratio of trapped particles is barely influenced by gravity.

The cluster identification method recalled in Sec. II B is applied to extract the distribution of the cluster volumes given in Fig. 3 (bottom). In agreement with the results obtained for 2D experimental data by Monchaux et al. ${ }^{13}$ the cluster sizes cover a large range of scales up to the integral scale and their distributions have algebraic right tails with exponent -2 regardless of the Stokes number and of the Rouse number. However, our simulation data exhibit a peak located toward the small scales that was absent in their PDFs. This discrepancy may be partly explained by the fact that their loading was such that the average distance between particles was of order $8 \eta$ while it is four times smaller in the present simulations. This peak can be interpreted as a maximum of preferential concentration occurring at flow scales of order $6 \eta$. It is interesting to notice that, by making use of the box counting method and of the radial distribution function respectively, Aliseda et al. ${ }^{2}$ and Wood et al. ${ }^{23}$ reported a maximal preferential concentration at flow scales of order $10 \eta$ consistently with our results.

To further analyse the role of gravity in the preferential concentration we show in Fig. 4 results collected for the 2D slices performed in planes perpendicular to gravity (dashed-dotted lines) and in planes along gravity (full lines). Figure 4(a) shows $\sigma_{\mathcal{V}}$ as a function of $S t$ for the four Rouse numbers. It appears that preferential concentration is slightly more important in planes along gravity. Even 

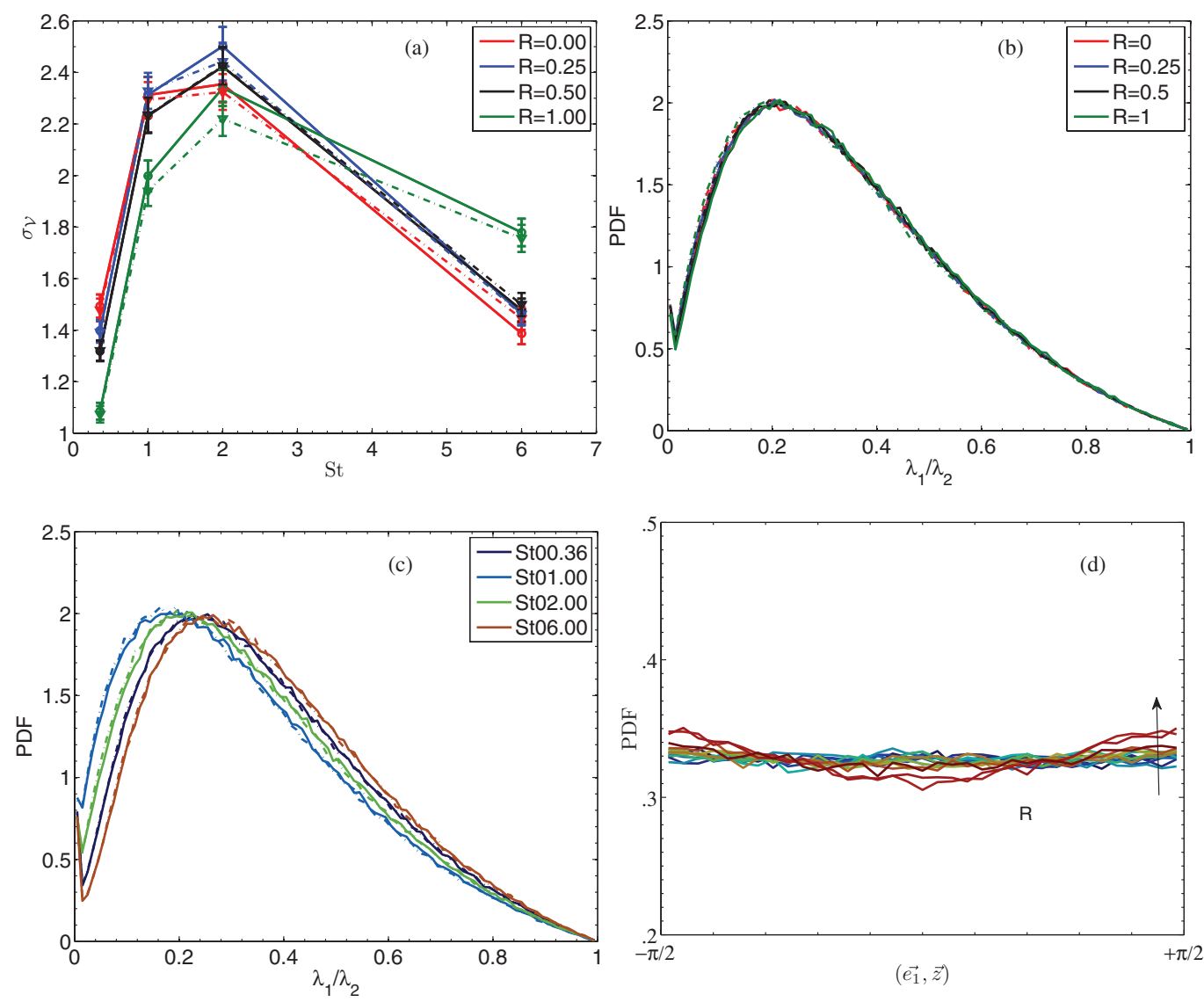

FIG. 4. Voronoï analysis on 2D slices. Standard deviation of the Voronoï areas as a function of $S t$ at fixed $R$ (a). PDFs of cell aspect ratio for $S t=2$ for each $R$ (b) and for $R=0.5$ for each $S t$ (c). PDFs of the angle between the Voronoï cells' principal axis of inertia $\vec{e}_{1}$ and the gravity direction $\vec{z}$ for each $(S t, R)$ values (d). When relevant, full line correspond to slices in planes containing gravity and dashed-dotted lines to planes perpendicular to gravity.

if the increase is less than 3\% (magnitude of the error bar), this effect is noticeable and evidences a slight anisotropy in the particle concentration field in presence of gravity, in particular for the largest value $R=1$. Figures 4(b) and 4(c) present the 2D Voronoï cells aspect ratio PDFs at fixed $R$ and $S t$. These PDFs do not depend at all on the plane orientation (full line and dashed-dotted line PDFs are almost superimposed). Consistently, it appears that gravity intensity does not influence much these PDFs shape while very elongated cells are more and more likely to be observed when $S t$ gets closer to unity. PDFs of the angle between the principal axis of inertia of the 2D Voronoï cells (or equivalently their elongated direction) and the gravity direction are shown in Fig. 4(d). As $R$ increases, these PDFs tend to be more and more bent with a most probable value approaching $\pm \pi / 2$, evidencing a tendency of the elongated cells to be perpendicularly oriented to gravity. For vanishing gravity and in planes containing gravity (not shown here) the PDFs are flat, meaning that no preferential orientation is found in these cases. Note that the effect of Rouse and Stokes numbers shown on the PDFs presented in Figure 4 was tracked in 3D data by performing a similar analysis. Effects were much less pronounced and not conclusive, maybe for statistical sample size problems.

\section{B. Settling velocity}

Figure 5 displays the PDFs of the particle centered vertical velocity $v_{z}$. PDFs were calculated for our three control parameters, namely, the Rouse number, the Stokes number, and the local concentration. They are plotted with two of these parameters fixed, the third being varied. We do not display all the possible combinations but rather a significant selection illustrating the main results. All PDFs are well described by Gaussian distributions. Gravity and local concentration have 

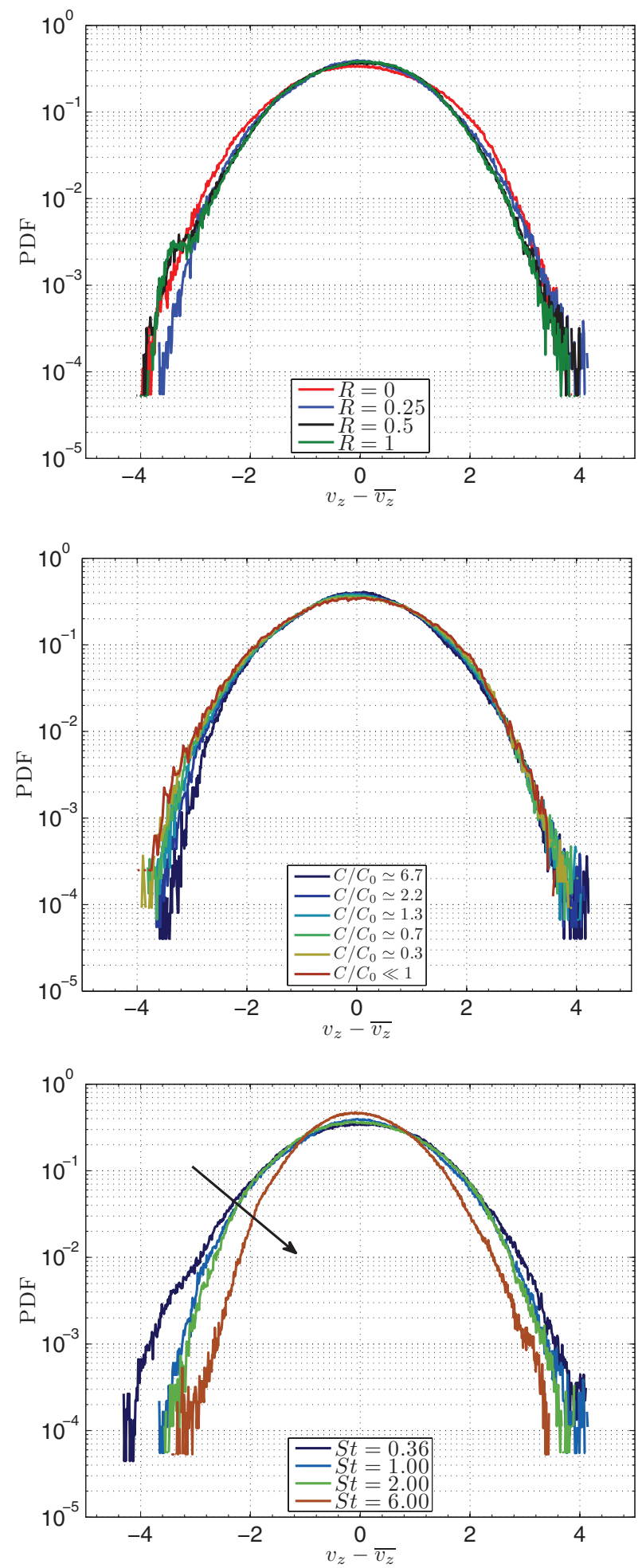

FIG. 5. PDF of the centered vertical velocity $v_{z}$ when varying the three control parameters independently (Stokes number, local concentration, and Rouse number). (Top) Rouse number is varying, $S t=1, C / C_{0} \simeq 2$. (Middle) Local concentration is varying, $S t=1, R=0.25$. (Bottom) Stokes number is varying, $R=0.25, C / C_{0} \simeq 2$. 

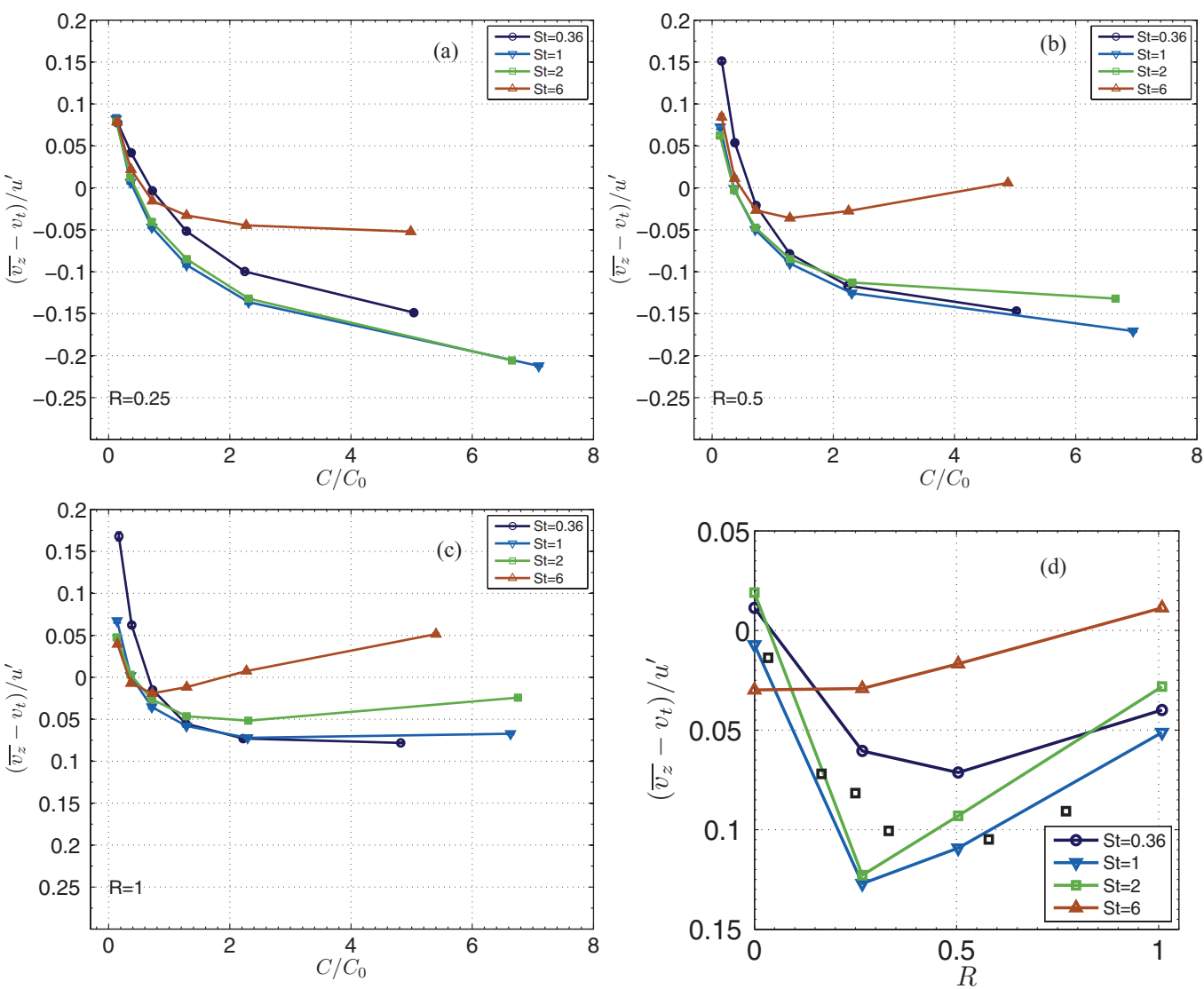

FIG. 6. (a)-(c) Conditional average of the settling velocity rate normalised by the turbulence intensity given as a function of the local concentration $C / C_{0}$; (d) Mean settling velocity rate given as a function of the Rouse number. Open squares corresponds to data from Yang and $\mathrm{Lei}^{24}$ for $R_{\lambda}=35.7, \mathrm{St}=1$.

negligible influence. Their standard deviation clearly diminishes when the Stokes number increases, a feature which is in agreement with a less intermittent behavior as the particle inertia becomes larger. Figure 5 also shows that the PDFs are slightly skewed to the left-hand side, reflecting the higher probability of the particle to settle faster than their mean descending velocity. This behavior is further analysed in Sec. III C.

The settling velocity rate, $\bar{v}_{z}-v_{t}$, normalized by $u^{\prime}$ is given as a function of the local concentration on Figs. 6(a)-6(c) for the four values of the Stokes number and of the Rouse number. A net increase of the settling velocity is found for all Stokes and Rouse numbers except when particle inertia and/or gravity become large $(S t=6$ and $R=1)$. At the very high local concentrations $\left(C / C_{0} \simeq 6-7\right)$ the settling rate reaches a maximum of $0.15 u^{\prime}-0.2 u^{\prime}$. Figure 2 indicates that this maximum involves a large amount of the particle sample, about $40 \%$ for $S t=1$ and 2 and up to $20 \%$ for $S t=0.36$ and 6. If we consider the mean settling rate as a function of the Stokes number and Rouse number (see Fig. 6(d)), we recover numerical and experimental results previously reported in the literature: ${ }^{22,24,26}$ a maximum enhancement of the settling velocity of order $0.1 u^{\prime}$ and occurring for Stokes numbers around unity and intermediate Rouse numbers $(R \simeq 0.25-0.5)$. We have added data from Yang and $\mathrm{Lei}^{24}$ obtained at $R_{\lambda}=35.7$ and $S t=1$ as an illustration. Note that for $R=0$, a nonvanishing settling velocity is measured that corresponds to statistical noise of order 0.03 . This value can be considered as an error bar for the points obtained at non-zero $R$ values.

\section{Fluid statistics}

The PDFs of the fluid acceleration, fluid velocity, and slip velocity at the particle position were calculated but are not shown. For each quantity, once center-reduced, the PDFs associated with 

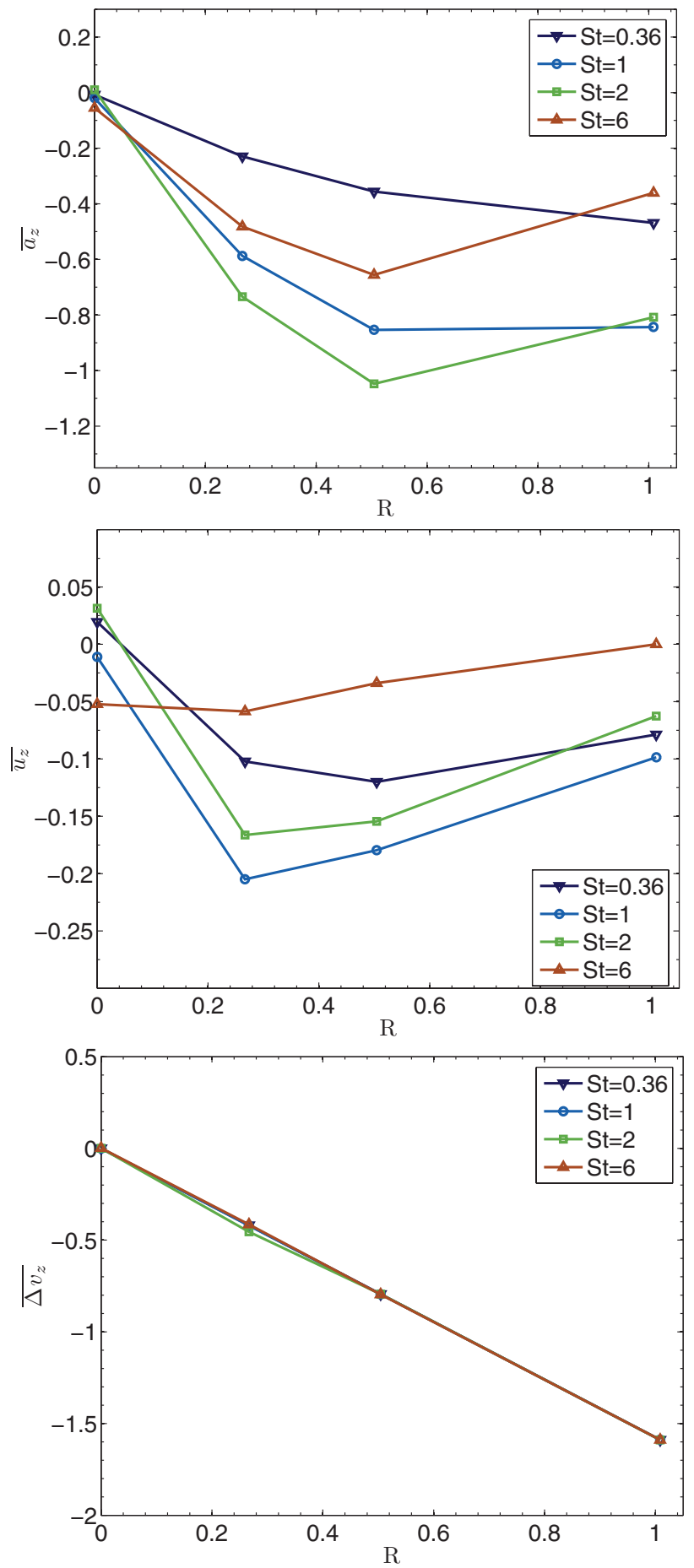

FIG. 7. Average values of vertical fluid acceleration (top), fluid velocity (middle), and slip velocity (bottom) at particle positions versus the Rouse number for iso values of the Stokes number.

the various flow parameters (Stokes number, Rouse number, and local concentration) were found to all collapse and can thus be fully described from their mean and standard deviation. The fluid acceleration displayed heavy tails that can be fitted with the now classical model due to Mordant et al. ${ }^{15}$ Gaussian distributions were obtained for the fluid velocity and the slip velocity PDFs were found roughly Gaussian with a little cusp at the origin. 


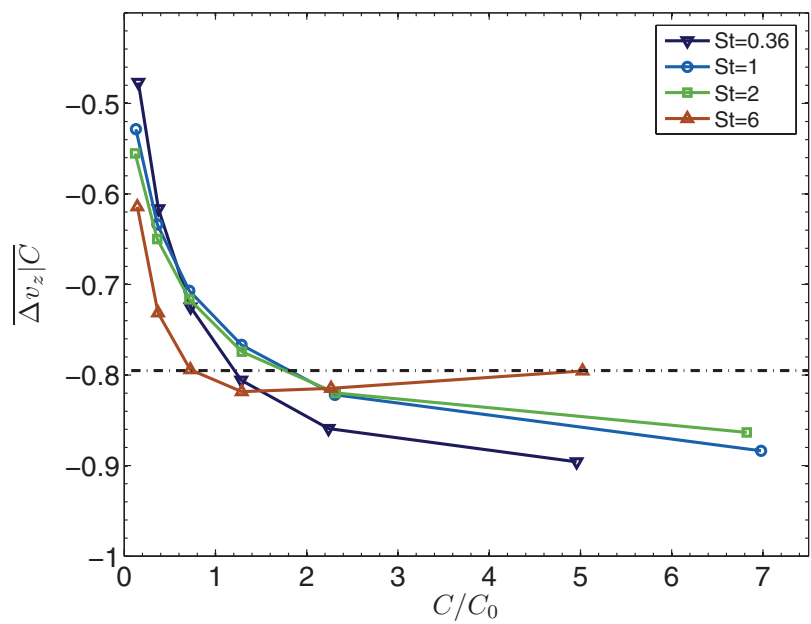

FIG. 8. Conditional average of $\overline{\Delta v_{z}}$ at the particles locations given as a function of the local concentration for the four values of the Stokes numbers and for $R=0.5$. The dashed line represents the value of terminal velocity $v_{t}$.

The mean values of the vertical fluid acceleration, $\overline{a_{z}}$, and vertical fluid velocity, $\overline{u_{z}}$, evaluated at the particle position are, respectively, presented in Figs. 7 (top) and 7 (middle) for the different Stokes and Rouse numbers considered. They show that, when gravity is turned on, particles tend to preferentially accumulate in regions of fluid acceleration and fluid velocity that are aligned with the gravity direction. This tendency is stronger for $S t \simeq 1-2$ and for $R \simeq 0.25-0.5$. The accumulation of particles in regions of descending flow velocity was previously reported by Wang and Maxey ${ }^{22}$ and is referred to as the preferential sweeping. This preferential sweeping is well illustrated by considering both $\bar{u}_{z}$ and the average slip velocity, $\overline{\Delta v_{z}}=\bar{v}_{z}-\overline{u_{z}}$, given in Figs. 7 (middle) and 7 (bottom) as a function of $R$. Both figures clearly show that the mean increase of the settling velocity comes from the local mean contribution of the fluid velocity since the average slip velocity exactly matches $v_{t}$ (this matching being a direct result from the linear model used to describe the drag force in Eq. (3)). However, if we consider now the mean of $\Delta v_{z}$ conditionally sampled at various local concentrations (see Fig. 8), it can be shown that particles settle faster than $v_{t}+u_{z}$ in the high concentration regions. This additional increase of the slip velocity results from the preferential concentration of particles along the downward fluid acceleration observed in Fig. 7 (top). Not shown

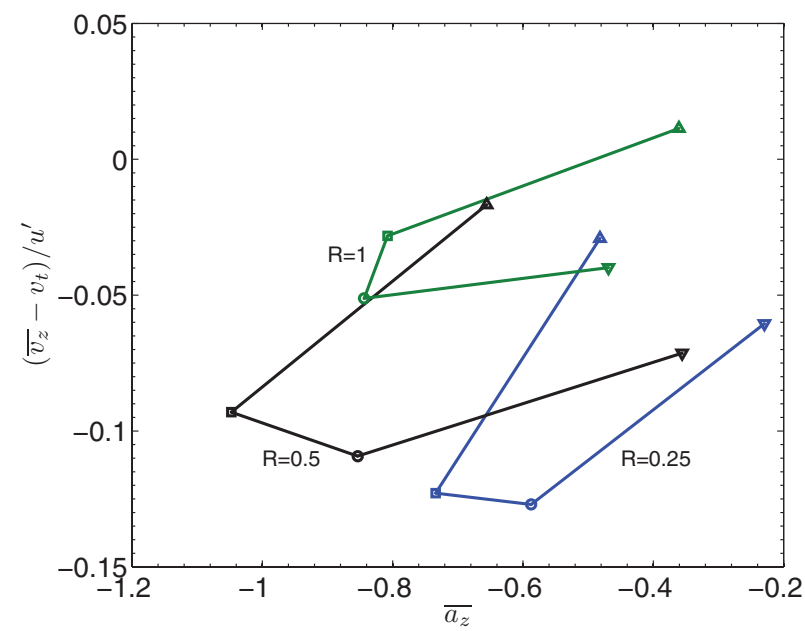

FIG. 9. Mean settling velocity rate given as a function of the mean fluid descending acceleration at particle positions. Symbols are $\nabla$ for $S t=0.36, \bigcirc$ for $S t=1, \square$ for $S t=2$, and $\Delta$ for $S t=6$. 

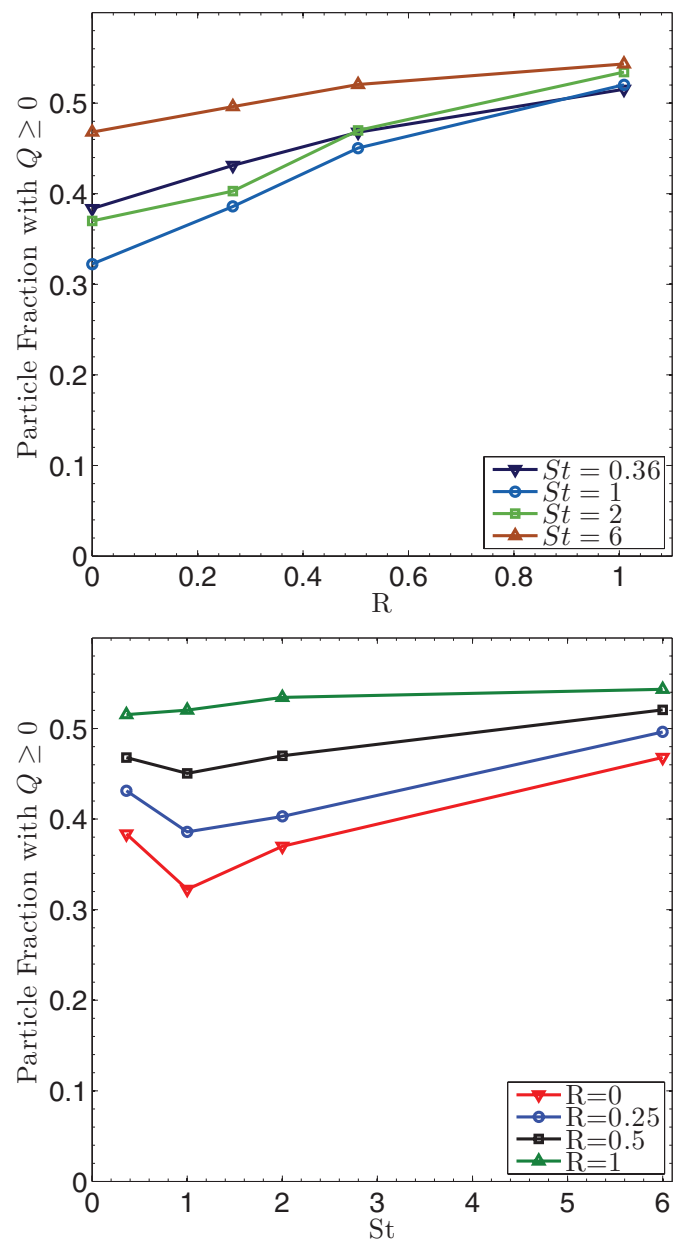

FIG. 10. Fraction of particles trapped in flow region with positive Okubo-Weiss parameter given as a function of the Rouse number (top) and of the Stokes number (bottom).

here but worth mentioning is that the fluid acceleration $a_{z}$ was found almost constant with the local concentration $C / C_{0}$ and to be mainly sensitive to the particle inertia and gravity as displayed in Fig. 7 (top). The preferential sampling of descending acceleration regions may act dynamically along the particle history and thus result in a global contribution to the settling enhancement which is not related to local (in time and space) concentration effects. This global contribution is well illustrated by Fig. 9 where we have represented the average settling velocity enhancement as a function of the fluid average descending acceleration given at the particle positions for the various Stokes numbers and at fixed $R$. The correlation between these two quantities is particularly important for $R=0.25$ and $R=0.5$. Note that this downward acceleration effect can explain the slight skewness of the vertical particle velocity PDFs previously observed in Fig. 5 (bottom), see Sec. III B.

Figure 10 presents the percentage of particles located in fluid regions characterized by a positive value of the Okubo-Weiss parameter, $Q$, defined as $Q=\frac{1}{2} \Omega_{i j} \Omega_{i j}-\frac{1}{2} S_{i j} S_{i j}$, where $S_{i j}=\frac{1}{2}\left(\partial u_{i} / \partial x_{j}+\partial u_{j} / \partial x_{i}\right)$ is the rate of strain tensor and $\Omega_{i j}=\frac{1}{2}\left(\partial u_{i} / \partial x_{j}-\partial u_{j} / \partial x_{i}\right)$ is the vorticity tensor, as a function of the Rouse number and of the Stokes number. It is interesting to observe that while more and more particles move from high-strain to high-vorticity fluid regions under increasing gravity effects, the minimum of the particle fraction, obtained for $S t=1$, remains present for a Rouse number as large as $R=0.5$. This suggests that the centrifuging of heavy particles away from high-vorticity regions dominates gravity when $R \leq 0.5$ while for $R \simeq 1$ centrifugal effects are less effective and overwhelmed by gravity. Nonetheless, Fig. 7 (middle) indicates that for $R=1$ 

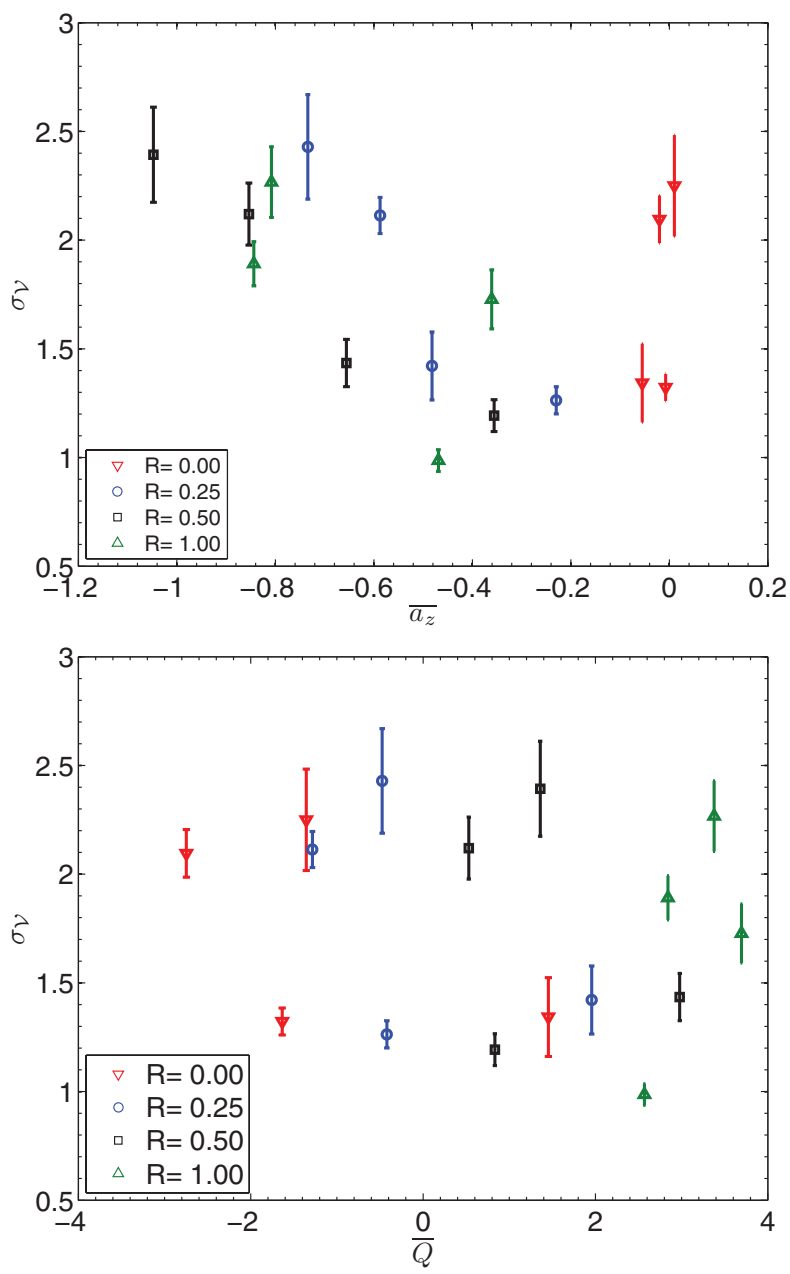

FIG. 11. Standard deviation of Voronoï volumes given as a function of the average vertical acceleration (top) and of the Okubo-Weiss parameter (bottom).

the average fluid velocity in regions of high particle concentration is well below zero (for $S t=0.36$, 1 , and 2), meaning that the preferentially sweeping mechanism is still efficient at this relative large value of gravity.

When gravity is turned on, the standard deviation of the Voronoï volumes exhibits a strong correlation with the vertical fluid acceleration in Fig. 11 (top): the larger is the descending acceleration and the larger $\sigma_{\mathcal{V}}$. On the other hand, no clear correlation between $\sigma_{\mathcal{V}}$ and the parameter $Q$ is observed in Fig. 11 (bottom). This infers the important role played by the fluid acceleration on the preferential concentration emphasized by Coleman and Vassilicos. ${ }^{4}$ In zero-gravity field, these authors found that concentration of heavy particles coincides with flow zones of zero-acceleration. They explained this by introducing the sweep-stick mechanism that involves the sweeping of turbulent small eddies by larger ones and causes the multiscale clustering of the spatial distribution of the particles. The correlation between $\sigma_{\mathcal{V}}$ and $a_{z}$ suggests that the stick part of this mechanism can be questionable under gravity effects.

\section{DISCUSSION AND CONCLUSIONS}

The Voronoï analysis given in Sec. III A, shows that the preferential concentration is mainly determined by the inertia of the particles and slightly altered by the gravity. As $R$ increases, the distribution of the particles tends to be somewhat more homogeneous, however this effect is still 
small for the Rouse number $R=1$, the largest value considered here. These findings agree well with Monchaux et al. ${ }^{13}$ who found similar results from the Voronoï analysis applied to experimental data that cover a range of Stokes numbers $0.2 \leq S t \leq 6$, of Rouse numbers $0.4 \leq R \leq 2$ and of Reynolds numbers $72 \leq R_{\lambda} \leq 114$. Thus, the physical mechanisms leading to preferential concentration of heavy particles appear to be robust under gravity effects even if the analysed global and local fluid and particle statistics have demonstrated that the preferential sampling is significantly altered by gravity. It is worth noticing that beside the centrifugal effects, other physical mechanisms give rise to highly nonuniform particle concentration: it is indeed possible to measure the same level of preferential concentration from a global quantity such as $\sigma_{\mathcal{V}}$ in different particulate-flow conditions for which the origin and/or the nature of the preferential concentration may differ from one flow to another. In this respect, the conditional statistics allow a more precise identification of the relevant mechanisms underlying particle clustering. Gravity may thus be expected to mostly influence the cluster shape and orientation and consequently the spatial structure of the clusters. The 2D Voronoï analysis presented in Fig. 4 shows that the Voronoï cells' shape is mostly governed by the Stokes number rather than by gravity. However, the particles tend to preferentially concentrate along the gravity direction. In addition, if the cell aspect ratio is found similar in zero and non-zero gravity fields, the cells tend to be perpendicularly oriented to gravity. It is observed that, in $3 \mathrm{D}$, clusters are actually elongated 2D manifolds. The present results show that these 2D manifolds (i) have the same structure regardless of the gravity intensity, (ii) are the most elongated for Stokes number around unity, and (iii) have a structure such that particles are closer to each others along the gravity direction. A study on the global structure of these manifolds-clusters would be very valuable, nevertheless, due to the difficulty of taking into account the periodicity of our data in the cluster identification process, we are not able to address this issue yet, but we consider it as an important challenge for the coming work. Note that our range of fluid and particle parameters makes that the particle Froude number, defined as $F r=\tau_{p} v_{t}^{2} /\left(u^{\prime 2} T\right)$ when based on the large scale flow (see Dávila and Hunt ${ }^{5}$ ), does not exceed 0.37 (for $R=1$ and $S t=6$ ). This means that the interaction between the fluid and particle inertial forces remains strong compared to the gravity force and in turn may partly explain the small effect of gravity reported here. However, increasing the Froude number implies to consider higher values of the Stokes number or of gravity, which is not suitable as the forcing method used in the simulations to maintain the turbulence stationary or the use of the periodic boundary conditions may alter the DNS results when $\tau_{p}$ becomes close to the large-eddy turn over time $T$ and/or when $R$ is well above unity.

The distribution of the cluster volumes displayed in Fig. 3 (bottom) corroborates the multiscale nature of clustering previously pointed out in Refs. 4,8, and 13. As noticed by Coleman and Vassilicos, ${ }^{4}$ at low Reynolds number, as the one considered herein, the centrifuging effects should be predominant in the clustering of particles while at a higher Reynolds number the effect of large coherent eddies should also be of importance on the particle segregation. This may partly explain the peak observed in Fig. 3 (bottom). Indeed, the position of this peak suggests that most of preferential concentration occurs at the dissipative flow scales where centrifuging effects are optimum. So, beside the difference between the particle loading used in the experiments and the present simulations (see Sec. III A), the absence of this peak in Monchaux et al. ${ }^{13}$ may be related to their higher Reynolds number flow. The Reynolds number dependence of the centrifuging and sweep-stick mechanisms on particle concentration would require a further study to be more conclusive.

The statistics provided on the fluid quantities reveal that centrifugal effects prevail gravity up to Rouse number $R \simeq 0.5$ and are small for $R \simeq 1$. Still, the non-homogeneities of the particle concentration remain important independently of the gravity field and a strong correlation between $\sigma_{\mathcal{V}}$ and the vertical fluid acceleration is observed (see Fig. 11). The fluid acceleration appears thus to effectively influence particle concentration under gravity effects in the low Reynolds number turbulent flow examined in the present simulations.

The average of the particle settling velocity conditioned on the local concentration shows that the settling velocity monotonically increases with the local concentration and is larger than its mean value in the high concentration regions of the flow. Note that, in a one-way coupling approach, this increase only results from the preferential sweeping that influences the trajectory of a large number of the sample particles (up to $40 \%$ in the most favourable case, see Fig. 2) and not from concentration 
effects that may alter the flow locally when a two-way coupling approach is used. As observed in Fig. 7, heavy particles tend to accumulate in regions of descending velocity and of descending acceleration. Thus, in addition to the fluid velocity contribution, the present analysis shows a further increase of the settling velocity by the fluid acceleration along the gravity direction. By taking into account the maximal contribution of both descending fluid velocity and fluid acceleration to the settling velocity enhancement, we found that the preferential sampling is optimum for $S t \simeq 1-2$ and for $R \simeq 0.25-0.5$.

Straightforward comparison of our results (Fig. 6) to those obtained experimentally by Aliseda et al. (see Figure 24 in Ref. 2) raises several comments. They found a linear increase of the settling velocity with the local concentration while the increase reported here is less than linear. This difference can be explained by the collective effects, i.e., the local double interaction between the flow and the particle clusters (not included herein), as suggested in their article. Note as well that, depending on the experimental conditions they considered, the settling rate velocity becomes positive for values of $C / C_{0}$ around 2.5-4 in contrast to $C / C_{o} \sim 1$ in our study. This latter value seems, however, more natural since the settling velocity enhancement results from the preferential concentration. Discussions with the authors evidenced that some biases on the actual measured value of the vertical velocity may remain. Their data could consequently be shifted, which may in turn partly explain the discrepancies between our results and theirs.

Finally, we want to outline that this work constitutes a first step in analyzing numerical data of heavy particles settling in a turbulent flow by making use of the Voronoï diagrams with the purpose of better understanding the mechanisms underlying the settling velocity enhancement. In a second step, we aim at going further on this topic by considering two-way coupling simulations that include mass loading effects.

\section{ACKNOWLEDGMENTS}

This work has been initiated at the COST-Action meeting "Particles in Turbulence" held in Potsdam in 2011. The organisers of this meeting are deeply acknowledged. We particularly thank Mickaël Bourgoin and Alain Cartellier for fruitful discussions on the topics addressed in this article. We also want to thank Alain Paris from ENSTA who has helped us in the process of sharing data which was the first important step of this collaboration. This study was partly supported by the Ramón y Cajal research program from the Spanish Ministry of Science.

${ }^{1}$ The different values of the upper and lower limits have been chosen as follows: 1 corresponds to the average value, 0.6 and 2 roughly correspond, respectively, to the lower and upper thresholds used for cluster and void identification. The values 0.3 and 4 are half and twice these latest values.

${ }^{2}$ A. Aliseda, A. Cartellier, F. Hainaux, and J. C. Lasheras, "Effect of preferential concentration on the settling velocity of heavy particles in homogeneous isotropic turbulence," J. Fluid Mech. 468, 77-105 (2002).

${ }^{3}$ J. Bec, A. Celani, M. Cencini, and S. Musacchio, "Clustering and collisions of heavy particles in random smooth flows," Phys. Fluids 17(7), 073301 (2005).

${ }^{4}$ S. W. Coleman and J. C. Vassilicos, "A unified sweep-stick mechanism to explain particle clustering in two- and threedimensional homogeneous, isotropic turbulence," Phys. Fluids 21, 113301 (2009).

${ }^{5}$ J. Dávila and J. C. R. Hunt, "Settling of small particles near vortices and in turbulence," J. Fluid Mech. 440, 117-145 (2001).

${ }^{6}$ J. R. Fessler, J. D. Kulick, and J. K. Eaton, "Preferential concentration of heavy particles in a turbulent channel flow," Phys. Fluids 6, 3742-3749 (1994).

${ }^{7}$ R. Gatignol, "The Faxen formulae for a rigid particle in an unsteady non-uniform Stokes flow," J. Mec. Theor. Appl 2 , 143-160 (1983).

${ }^{8}$ S. Goto and J. C. Vassilicos, "Self-similar clustering of inertial particles and zero-acceleration points in fully developed two-dimensional turbulence," Phys. Fluids 18(11), 115103 (2006).

${ }^{9}$ J. Jímenez and A. A. Wray, "On the characteristics of vortex filaments in isotropic turbulence," J. Fluid Mech. 373, 255-285 (1998).

${ }^{10}$ M. R. Maxey and J. J. Riley, “Equation of motion for a small rigid sphere in a nonuniform flow,” Phys. Fluids 26, 883-889 (1983).

${ }^{11}$ E. Meneguz and M. W. Reeks, "Statistical properties of particle segregation in homogeneous isotropic turbulence," J. Fluid Mech. 686, 338-351 (2011).

${ }^{12}$ R. Monchaux, "Measuring concentration with Voronoï diagrams: Study of possible biases," New J. Phys. 14, 095013 (2012). 
${ }^{13}$ R. Monchaux, M. Bourgoin, and A. Cartellier, "Preferential concentration of heavy particles: A Voronoï analysis," Phys. Fluids 22, 103304 (2010).

${ }^{14}$ R. Monchaux, M. Bourgoin, and A. Cartellier, "Analyzing preferential concentration and clustering of inertial particles in turbulence," Int. J. Multiphase Flow 40, 1-18 (2012).

${ }^{15}$ N. Mordant, A. M. Crawford, and E. Bodenschatz, "Three-dimensional structure of the Lagrangian acceleration in turbulent flows," Phys. Rev. Lett. 93(21), 214501 (2004).

${ }^{16}$ W. C. Reade and L. R. Collins, "Effect of preferential concentration on turbulent collision rates," Phys. Fluids 12, 2530-2540 (2000).

${ }^{17}$ R. Rogallo, "Numerical experiments in homogeneous turbulence," NASA Tecn. Report 81315, 1981.

${ }^{18}$ P. G. Saffman and J. S. Turner, "On the collision of drops in turbulent clouds," J. Fluid Mech. 1, 16-30 (1956).

${ }^{19}$ J. P. L. C. Salazar, J. De Jong, L. J. Cao, S. H. Woodward, H. Meng, and L. R. Collins, "Experimental and numerical investigation of inertial particle clustering in isotropic turbulence," J. Fluid Mech. 600, 245-256 (2008).

${ }^{20}$ K. D. Squires and J. K. Eaton, "Preferential concentration of particles by turbulence," Phys. Fluids 3, 1169-1178 (1991).

${ }^{21}$ Y. Tagawa, J. Martinez Mercado, V. N. Prakash, E. Calzavarini, C. Sun, and D. Lohse, "Three-dimensional Lagrangian Voronoï analysis for clustering of particles and bubbles in turbulence,” J. Fluid Mech. 693, 201-215 (2012).

${ }^{22}$ L. P. Wang and M. R. Maxey, "Settling velocity and concentration distribution of heavy particles in homogeneous isotropic turbulence," J. Fluid Mech. 256, 27-68 (1993).

${ }^{23}$ A. M. Wood, W. Hwang, and J. K. Eaton, "Preferential concentration of particles in homogeneous and isotropic turbulence," Int. J. Multiphase Flow 31, 1220-1230 (2005).

${ }^{24} \mathrm{C}$. Y. Yang and U. Lei, "The role of the turbulent scales in the settling velocity of heavy particles in homogeneous isotropic turbulence,” J. Fluid Mech. 371, 179-205 (1998).

${ }^{25}$ T. S. Yang and S. S. Shy, "The settling velocity of heavy particles in an aqueous near-isotropic turbulence," Phys. Fluids 15, 868-880 (2003)

${ }^{26}$ T. S. Yang and S. S. Shy, "Two-way interaction between solid particles and homogeneous air turbulence: Particle settling rate and turbulence modification measurements," J. Fluid Mech. 526, 171-216 (2005). 